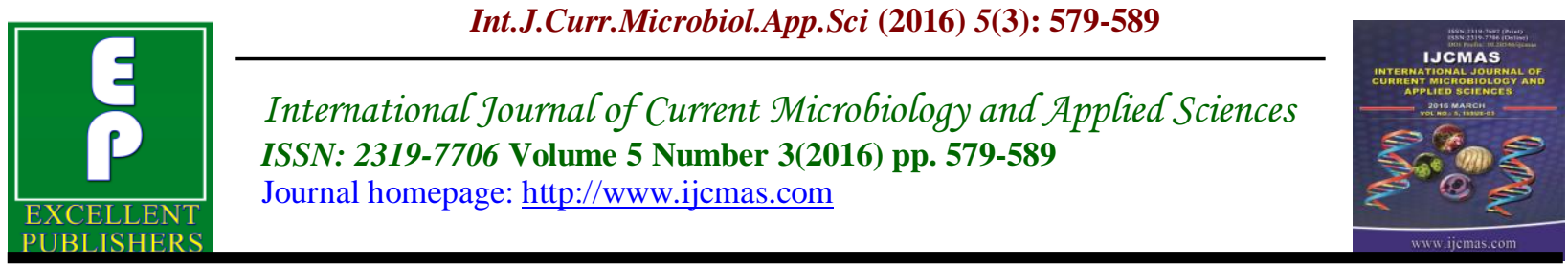

Original Research Article

http://dx.doi.org/10.20546/ijcmas.2016.503.067

\title{
A Comparative Analysis on Identification and Antifungal Susceptibility Profile of Candida Isolates Using Conventional and Automated Techniques
}

\author{
S. Prasanna ${ }^{1}$, Partha Roy ${ }^{2}$, Naveen Grover ${ }^{3}$, Puneet Bhatt ${ }^{4}$, \\ Dudhat Vaibhav Laxmikant Rao ${ }^{5}$ and Nikunja Kumar Das ${ }^{6}$ \\ ${ }^{1}$ Department of Microbiology, Shri Sathya Sai Medical College and Research Institute, \\ Ammapettai, Kancheepuram-608103, India \\ ${ }^{2}$ Department of Microbiology, Delhi Base Hospital, Delhi, India \\ ${ }^{3}$ Department of Microbiology, AFMC, Pune-411040, India \\ ${ }^{4}$ Department of Microbiology, CH (SC), Pune-411040, India \\ ${ }^{5}$ Consultant Microbiologist, Sayadhri Hospital, Pune, India \\ ${ }^{6}$ Department of Microbiology, D.Y.Patil Medical College, Pune, India \\ *Corresponding author
}

\begin{tabular}{|c|c|}
\hline & A B S T R A C T \\
\hline & \multirow{7}{*}{$\begin{array}{l}\text { Candida species are the most common fungal pathogen isolated from clinical } \\
\text { samples. Of late, improper use of antifungal drugs in critically ill patients has } \\
\text { favoured antifungal drug resistance amongst Candida. To isolate and identify } \\
\text { Candida species from hospitalized patients and determine their antifungal } \\
\text { susceptibility profile by conventional (Epsilometer i.e., E-test) and automated } \\
\text { (VITEK-2) methods. This cross sectional study was carried out from December } \\
2012 \text { to June } 2014 \text { from hospitalized patients at a tertiary care centre, in which } 50 \\
\text { non-repetitive clinical isolates of Candida were analysed. The identification was } \\
\text { carried by conventional methods as per standard laboratory protocols and by } \\
\text { VITEK-2 Compact (Biomerieux, France). The antifungal susceptibility testing } \\
\text { (AFST) of these isolates was carried out by E-test and VITEK-2 Compact (YST } \\
\text { cards- AST YSO1). Out of } 50 \text { isolates, } 36 \text { ( } 72 \% \text { ) were non-albicans Candida and } 14 \\
\text { ( } 28 \% \text { were C.albicans. AFST by VITEK-2 showed that only } 2 \text { isolates ( } 4 \% \text { ) were } \\
\text { resistant to fluconazole and E-test showed that } 24 \text { isolates ( } 48 \% \text { ) were resistant to } \\
\text { fluconazole and voriconazole but all were sensitive to amphotericin B. There was } \\
\text { substantial agreement of } 80 \% \text { ( } 95 \% \text { CI of } 69-91 \% \text { ) for identification and less than } \\
\text { chance agreement for AFST between VITEK-2 and E-test. We conclude that E- } \\
\text { test can be a simple and convenient method for AFST when compared to VITEK-2. } \\
\text { We cannot rely only on VITEK- } 2 \text { for AFST. Identification and AFST of Candida } \\
\text { isolates must be carried out for optimum patient care. }\end{array}$} \\
\hline & \\
\hline Candida species, & \\
\hline $\begin{array}{l}\text { Antifungal } \\
\text { susceptibility } \\
\text { testing, } \\
\text { VITEK-2, E-test. }\end{array}$ & \\
\hline Article I & \\
\hline & \\
\hline & \\
\hline
\end{tabular}

\section{Introduction}

Candida speciesare part of the normal flora of skin, gut and genitals and is capable of causing a variety of infections and is emerging as an important nosocomial pathogen. They are the most common cause of fungal infections leading to invasive life 
threatening diseases. At present, candidal infections are the fourth leading cause of blood stream infections and one of the common opportunistic pathogen $(1,2)$. The increasing incidence of HIV infections, widespread use of antibiotics, organ transplantation, use of immunosuppressive agents and emergence of resistance to antifungal agents has contributed to the increased incidence of Candida infections.

The increasing incidence of invasive candidiasis in critically ill patients in ICU, acute wards, oncology wards and resistance to antifungal drugs is becoming worrisome.Long term use of azoles in the prophylaxis of systemic fungal infections in bone marrow transplant patients and for long-term suppressive therapy in patients with AIDS is an important factor in the selection of isolates that exhibit increased resistance to azole therapy $(3,4)$.

Till recent times,Candida albicans was considered as the most frequently isolated Candida species but non-albicans Candida have now become predominant $(5,6,7)$. The commonly isolated non-albicans Candidaare C. tropicalis, C.parapsilosis, C. guilliermondii, C. dubliniensis, C. glabrata, C.lusitaniae, C.kefyr and C.krusei. Some of the non-albicans Candida are intrinsically resistant to fluconazole (C.krusei) and some of them show resistance through enzyme modification to fluconazole (C.glabrata) $(6,7,8,9,10)$. In view of this, reporting the infecting species of Candida alongwith antifungal susceptibility testing is of utmost importance. We studied the prevalence of various Candida species in hospitalized patients at a tertiary care centrealong with their antifungal susceptibility pattern to commonly used antifungal drugs by two methods namely, E-test, a conventional method and secondly by VITEK-2, an automated system $(5,9,10)$.

\section{Materials and Methods}

\section{Sampling and Identification}

A cross sectional study was carried out from Dec 2012 to Jun 2014, in which a total of 50 non-repetitive clinical isolates of Candida were included from various clinical samples like blood, urine, muco-cutaneous samples (nail clippings, oral scrapings and high vaginal swabs), pleural fluids and continuous ambulatory peritoneal dialysis (CAPD) fluids. The blood samples were collected in BACTEC (BD BACTEC Mycosis IC/F, Ireland) and brain heart infusion (BHI) bottlesunder aseptic precautions. Clean catch midstream urine, urine from catheter, body fluids and CAPD dialysate were collected in wide mouthed sterile universal containers. Samples were transported and processed immediately as per standard laboratory protocols.

The organism was identified using conventional methods that included Gram stain,colony morphology in Sabouraud dextrose agar (SDA) with and without chloramphenicol. Germ tube test was used to differentiate C.albicans from nonalbicans Candida. CHROM agar (HI media) inoculation was done for differentiating the Candida species exhibiting differently coloured colonies, example: C.albicanslight green colour, C.tropicalis- dark blue colour, C.krusei- rough pink colour, C.glabrata- smooth pink colour, C.parapsilosis- creamy white to pale pink, C.dubliniensis- dark green(Fig 1). The sugar fermentation test was carried out using $2 \%$ sugars (Glucose, sucrose, maltose, trehalose and lactose). The sugar assimilation test was carried out with eight different sugar discs of $2 \%$ concentration (Glucose, sucrose, maltose, lactose, trehalose, xylose, cellobiose and dulcitol) in yeast nitrogen base agar (HI media) which is a 
carbohydrate deficient medium and the test candida strains were inoculated by pour plate method (Fig 2). Cornmeal agar with Tween-80 inoculation (HI media) were done in the Dalmau plates which is a nutrient deficient medium, used for observing sporulation and formation of blastoconidia, pseudohyphae and chlamydospore under 10x and 40x magnification (Fig 3). These conventional methods are the gold standard for identification of the candida in this study. ATCC Candida strains were procured from PGI Chandigarh and used as controlsCandida parapsilosis (ATCC 22019); Candida krusei (ATCC 6258); Candida albicans (ATCC 90028)

Identification of yeast and yeast like organisms was simultaneously carried out by automated system [VITEK-2 Compact (Biomerieux, France)] using VITEK-2 cards (ID-YST cards)in parallel. The ID-YST cards used for yeast identification consist of two cassettes or cards. The first card is for identification (ID card) that consists of various sugars for assimilation and biochemical reactions in small reaction tubes. The final identification was done by matching with the yeast data library in the VITEK-2 systems software in the form of binary digit representation like 1 (positive, + ) or 0 (negative, -). The second card is for yeast susceptibility testing (YST card).

\section{Antifungal Susceptibility Testing (AFST)}

Antifungal susceptibility testing (AFST) was carried out by conventional method (E-test) and automated method (VITEK-2).

E-test:A 0.5 McFarland standard suspension of Candida strain was prepared and E-strips (AB Biodisk, Solna, Sweden) of amphotericin B, voriconazole and fluconazole were applied over the RPMI 1640 agar with L-glutamine but without sodium bicarbonate (HI media) at $\mathrm{pH}$ 7.0. MIC of fluconazole, voriconazole and amphotericin B was determined.The zone edge intersecting the graded strip at the minimum concentration of the antibiotic was interpreted as MIC (Fig 4 \& 5). All the results were interpreted according to CLSI M27-S4 (2012)(17).

VITEK-2:A 2.0 McFarland standard suspension of Candida strain was prepared and yeast susceptibility testing (YST) card was used for AFST i.e., AST-YS01 kits. This card or cassette consists of following antifungal powders (5-flucytosine, fluconazole, voriconazole, amphotericin B and caspofungin) in small reaction tubes. Minimum inhibitory concentration (MIC) of 5-flucytosine, fluconazole, voriconazole, amphotericin B and caspofungin was determined by VITEK-2 Compact (Biomerieux, France).The results provided by VITEK-2 were interpreted as sensitive, intermediate or resistant for that particular antifungal drug, depending upon the yeast identified and by correlating the MIC values with CLSI and EUCAST guideline installed in VITEK-2systems software. But VITEK-2 does not give the result as per the break point levels for each antifungal drug and the exact MIC value is not determined.

\section{Statistical Analysis}

The concordance between conventional and automated methods regarding identification and antifungal susceptibility testing (AFST) of Candida isolates was measured by kappa co-efficient using online software available at http:/graphpad.com/ quickcalcs/ kappa1.cfm. as follows:(22)

(a) Less than chance agreement: $<0$

(b) Slight agreement: 0.01-0.20 (1-20\%) 
(c) Fair agreement: 0.21-0.40 (21-40\%)

(d)Moderate agreement: 0.41-0.60 (41-60\%)

(e) Substantial agreement: $0.61-0.80$ (61$80 \%)$

(f) Almost perfect agreement: 0.81-0.99 (81$99 \%)$

\section{Results and Discussion}

In this study, the most common sample from which Candida species were isolated was urine (24) followed by blood (13), mucocutaneous specimens (08) and other body fluids (05) such as pleural and CAPD fluids (Fig 6). The 36 out of 50 isolates were nonalbicans Candida (C.tropicalis- 21, C.parapsilosis- 11, C.glabrata - 02, C.guilliermondii- 01, C.kefyr- 01) and the rest 14 were $C$. albicans(Fig 7).All isolates exhibited gram positive budding yeast forms with pseudohyphae except C.glabrata (budding yeast forms only). The germ tube test effectively differentiated C.albicans from non-albicans Candida. The CHROM agar inoculation showed different coloured colonies depending on the species. The sugar fermentation and assimilation reactions and cornmeal agar with Tween-80 inoculation differentiates between different candida species.

Comparison between conventional method and automated method (VITEK-2) in identification was done. Out of 50 isolates, 21 were identified as C.tropicalis by conventional method whereas 22 as C.tropicalis by VITEK-2. A total of 14 isolates were identified as C.albicans by conventional method whereas VITEK-2 identified only 09 as C.albicans. Conventional methods identified 11 isolates as C.parapsilosis whereas VITEK-2 identified 09 as C.parapsilosis. Two
C.parapsilosis isolate were misidentified as C.lusitaniae and C.tropicalis by VITEK-2. Similarly C.albicans, C. glabrata and C.guilliermondii were misidentified as $C$. famata by VITEK-2 Compact. So between two methods, 10 out of 50 isolates showed discrepancy in identification (Table 1).

AFST was done for 50 isolates by both VITEK-2 and E-test method, out of which VITEK-2 identified only 02 isolates as resistant to fluconazole (C.guilliermondii and C.lusitaniae), whereas, 24 isolates were found to be resistant to fluconazole and voriconazole and all isolates were sensitive to amphotericin B (Fig $6 \&$ 7) by E-test method. Out of these 24 resistant isolates, 14 were C.tropicalis, 05 were C.albicans, 04 were C.parapsilosis and 01 was C.glabrata (Table 2). The results of minimum inhibitory concentration (MIC) values were interpreted as per CLSI M27-S4 (2012) (16).

\section{The Statistical Analysis}

Statistical analysis was carried out for conventional and automated methods, which showed "Substantial agreement" of $80 \%$ (95\% CI of $69-91 \%$ ) in identification of 40 out of 50 isolates. Ten discrepant isolates were therefore excluded for calculation of agreement and confidence interval (CI) for antifungal susceptibility testing. Two isolates which showed fluconazole resistance by VITEK-2 were sensitive by Etest. Twenty four isolates (48\%) were found to be resistant to fluconazole and voriconazole by E-test, whereasVITEK2identified all of them as sensitive. Therefore, there was no agreement ("less than chance agreement") between VITEK-2 and E-test for antifungal susceptibility testing (22).

Candida species, previously considered a harmless colonizer, have now emerged as 
significant pathogens. They are considered as the most common opportunistic fungus. Candida species are the most common cause of fungal infections leading to invasive life threatening diseases and it is the fourth leading cause of blood stream infections $(2,5,6)$. Candida colonizes the GI tract of more than $90 \%$ of healthy humans. C.albicans earlier accounted for majority of isolates in clinical samples, but in recent years non-albicans Candida have become more common, probably because of their greater antifungal drug resistance $(6,7,8)$.Isolates of C.albicans were predominantly sensitive to amphotericin B when compared to non-albicans Candida despite the use of polyene antifungal drugs for over five decades. The most common nosocomial infection caused by Candida is urinary tract infection (associated with instrumentation and catheterization) followed by blood stream infections, oral thrush and skin infections.

The increasing incidence of HIV infections, wide spread use of antibiotics, organ transplantation and use of immunosuppressive agents continue to be important factors responsible for the increasing incidence of Candida infections. Furthermore, Candida has assumed greater clinical importance because of their increasing resistance to various antifungal agents $(3,4,5,6,7)$.Some of the non-albicans Candida are intrinsically resistant to fluconazole (C.krusei) and some are resistant to fluconazole due to enzyme modification (C.glabrata). Therefore, reporting the infecting species of Candida along with antifungal susceptibility pattern must be carried out for optimum patient care and outcome $(6,7,8,9,10)$.

This study had a sample size of 50 Candida isolates with a distribution of $24(48 \%)$ from urine, $13(26 \%)$ from blood, 08 (16\%) from muco-cutaneous specimens and 05 (10\%) from other body fluids (Pleural and CAPD fluids). A study carried out on 102 patients with candidiasis at New Delhi also obtained similar sample distribution with predominant sample being urine followed by blood and oral scrapings (11).

Out of 50 isolates, $36(72 \%)$ were non albicans Candida and 14 (28\%) were C.albicans. So the prevalence of nonalbicans Candida was more when compared to C.albicans. This observation is in consonance with the changing trend seen both in India and across the world. Rani et al(2002), Chakrabarti A et al(2002), Agarwal et al (2004), Capoor et al(2005)and Krcmery V et al (2002) also showed similar shift from C.albicans to non-albicans Candida whereas Wingard et al (1995) found Candida albicans as the predominant isolates $(6,7,8,9,10,11,12,13)$.

In this study, among the non-albicans Candida, C.tropicalis (42\%) was the most commonly isolated species followed by C.parapsilosis $(22 \%)$ and others $(8 \%)$ which includes C.glabrata(4\%), C.guilliermondii (2\%) and C.kefyr $(2 \%)$.

Other studies carried out in India also reported C.tropicalis to be the predominant non-albicans Candida $(8,9,10,11,12)$. In this study, 06 out of 50 isolates (C.glabrata- 02 , C.albicans- $\quad 02, \quad$ C.kefyr- 01 , C.guilliermondii-01) were misidentified as C.famata by VITEK-2. Studies carried out by Jensen et al (2011) and Castanheira et al (2013)also reported similar misidentification of C.albicans, C.glabrata and C.guilliermondii as C.famataby VITEK$2(14,15)$. The correct identification of Candida species assumes importance not only for epidemiological purposes but also for management due to variation in antifungal susceptibility. 
Table.1 Discordance in Identification between Conventional and Automated Method (VITEK-2)

\begin{tabular}{|l|c|c|}
\hline Candidaspecies & Conventional methods & $\begin{array}{c}\text { Automated method } \\
\text { (VITEK-2) }\end{array}$ \\
\hline Candida tropicalis & 21 & 22 \\
\hline Candida albicans & 14 & 09 \\
\hline Candida parapsilosis & 11 & 09 \\
\hline Candida famata & 00 & 06 \\
\hline Candida guilliermondii & 01 & 03 \\
\hline Candida glabrata & 02 & 00 \\
\hline Candida kefyr & 01 & 00 \\
\hline Candida lusitaniae & 00 & 01 \\
\hline
\end{tabular}

Table.2 Discordance in Anti-Fungal Susceptibility Testing between Conventional Method (ETest) and Automated Method (Vitek-2) Out of Total 50 Isolates

\begin{tabular}{|l|c|c|c|c|}
\hline Candidaspecies & \multicolumn{2}{|c|}{ Sensitive isolates } & \multicolumn{2}{c|}{ Resistant isolates } \\
\hline $\begin{array}{l}\text { Total Candida species (50) by } \\
\text { Conventional method }\end{array}$ & $\begin{array}{c}\text { Conventiona } \\
\text { l methods } \\
\text { (E-test) }\end{array}$ & $\begin{array}{c}\text { Automated } \\
\text { method } \\
\text { (VITEK-2) }\end{array}$ & $\begin{array}{c}\text { Conventiona } \\
\text { l methods } \\
\text { (E-test) }\end{array}$ & $\begin{array}{c}\text { Automated } \\
\text { method } \\
\text { (VITEK-2) }\end{array}$ \\
\hline Candida tropicalis (21) & 07 & 22 & $\mathbf{1 4}$ & 00 \\
\hline Candida albicans (14) & 09 & 09 & $\mathbf{0 5}$ & 00 \\
\hline Candida parapsilosis (11) & 07 & 09 & $\mathbf{0 4}$ & 00 \\
\hline Candida famata & 00 & 06 & 00 & 00 \\
\hline Candida guilliermondii (01) & 01 & 02 & 00 & $\mathbf{0 1}$ \\
\hline Candida glabrata (02) & 01 & 00 & $\mathbf{0 1}$ & 00 \\
\hline Candida kefyr (01) & 01 & 00 & 00 & 00 \\
\hline Candida lusitaniae & 00 & 00 & 00 & $\mathbf{0 1}$ \\
\hline
\end{tabular}

Fig.1 CHROM Agar Inoculation for Different Candida species Showing- Light Green Colour (C.albicans); Bluecolour (C.Tropicalis) and Cream to Pale Pink Colour (C.parapsilosis)

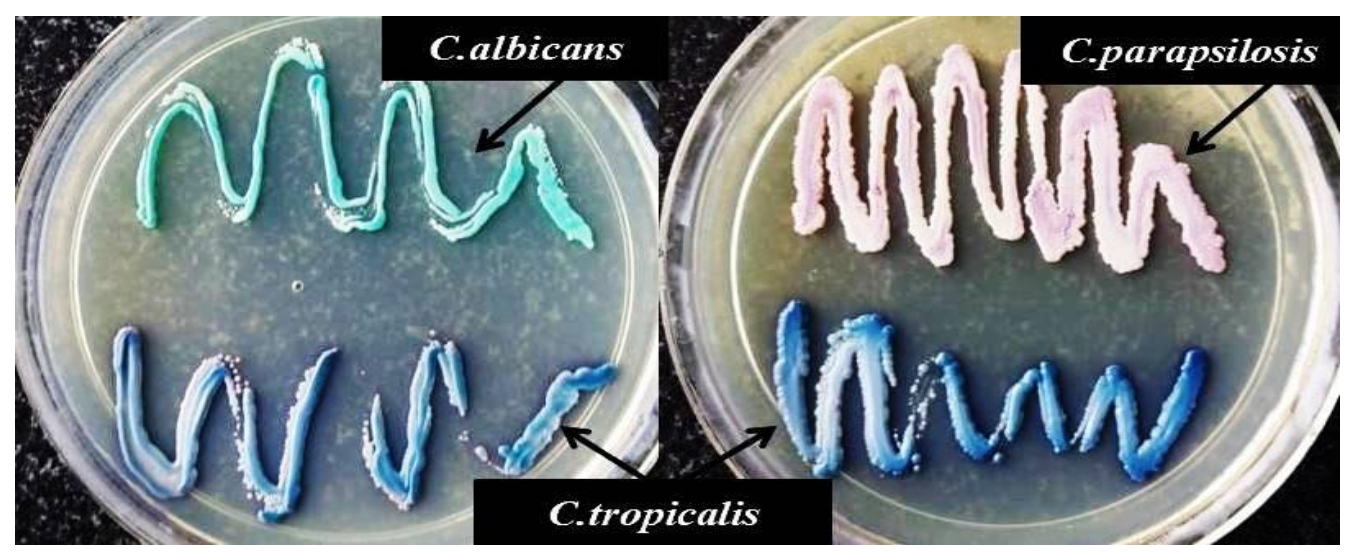


Fig.2 Sugar Assimilation Test (Indicator Bromocresol Purple) by Pour Plate Method for Eight Different Sugars for C.albicans. Yellow Discoloration and Growth Around Glucose, Maltose, Sucrose and Trehalose Indicates a Positive Assimilation Test

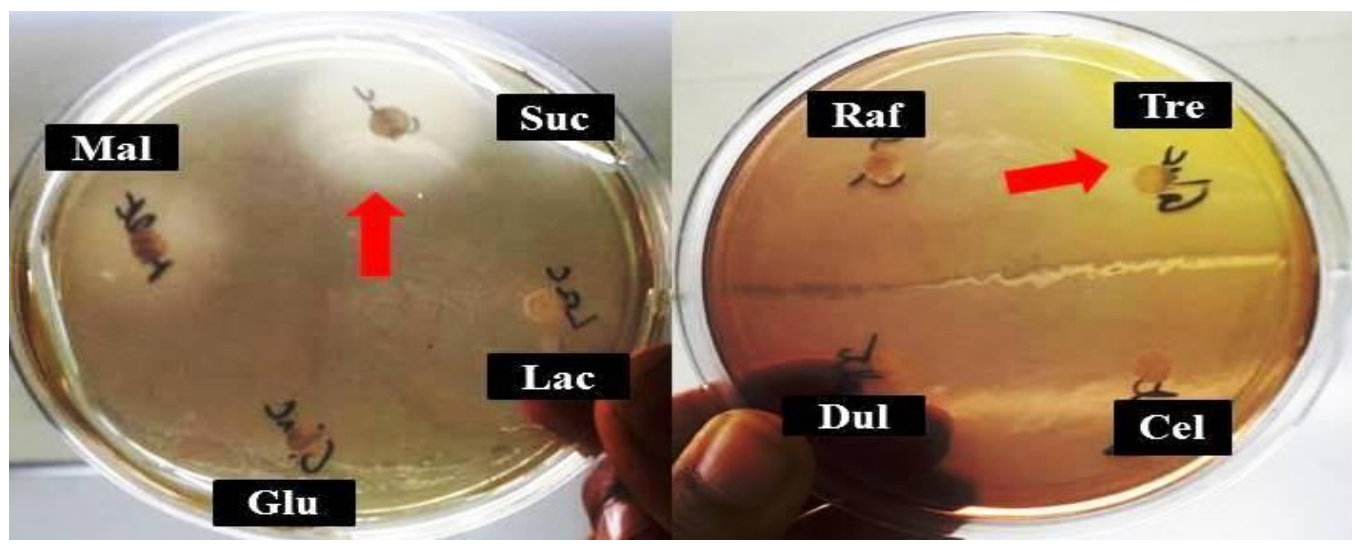

Fig.3 Cornmeal Agar with Tween-80 Inoculation for Candida species: 1. C.albicans Showing Branching Pseudo Hyphae with Bunch of Blastoconidia and Terminal Single Chlamydospores. 2. C.glabrata- Budding Yeast without Pseudo Hyphae. 3. C.parapsilosis- Abundant Branched Pseudo Hyphae (Tree Like Pattern) with Few Blastoconidia. 4. C.tropicalis- Long Pseudo Hyphae with Numerous Ovoid blastoconidia

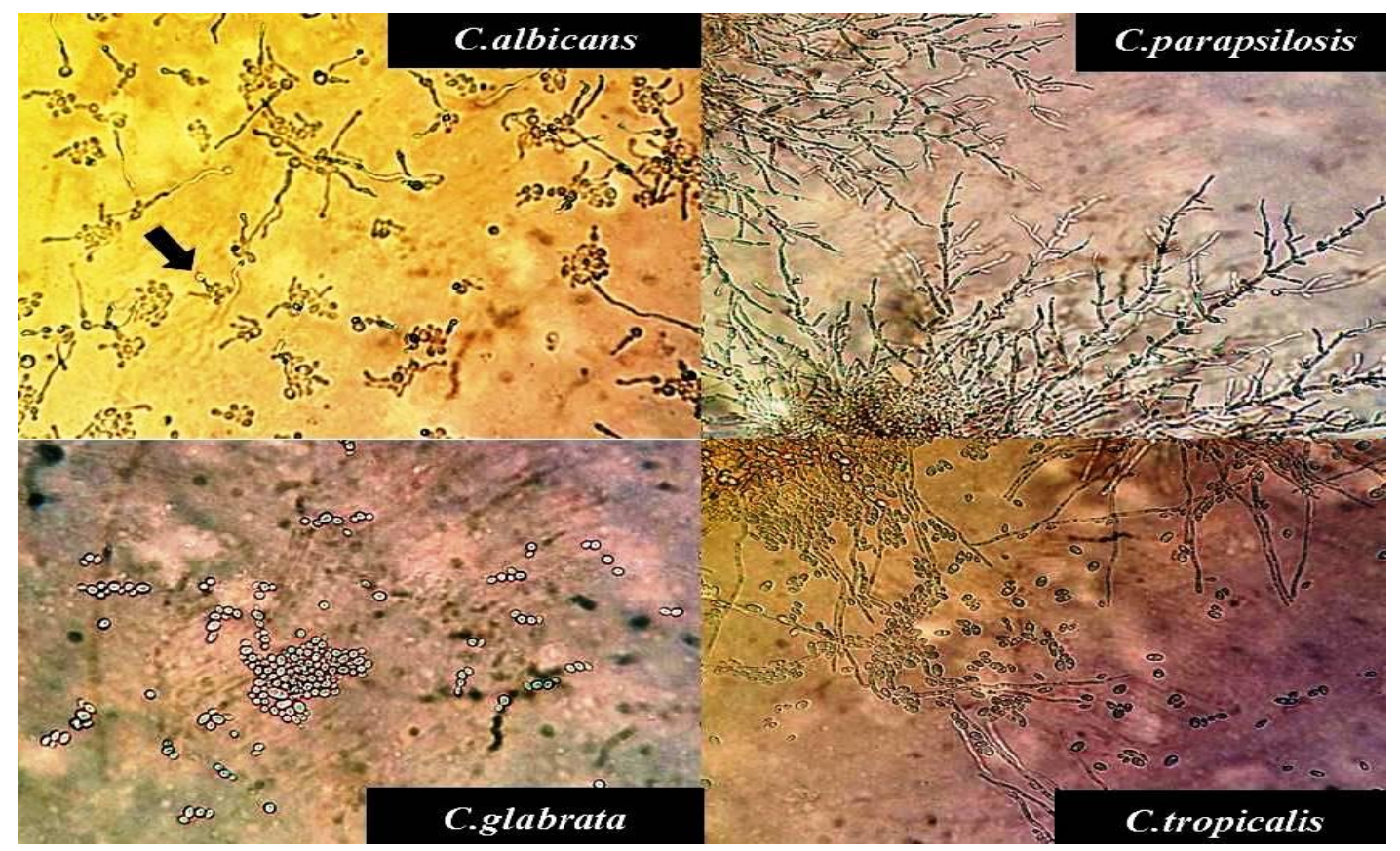


Fig.4 E-Test Method for AFST: Sensitive to Fluconazole and Amphotericin B (left) and Voriconazole (right)

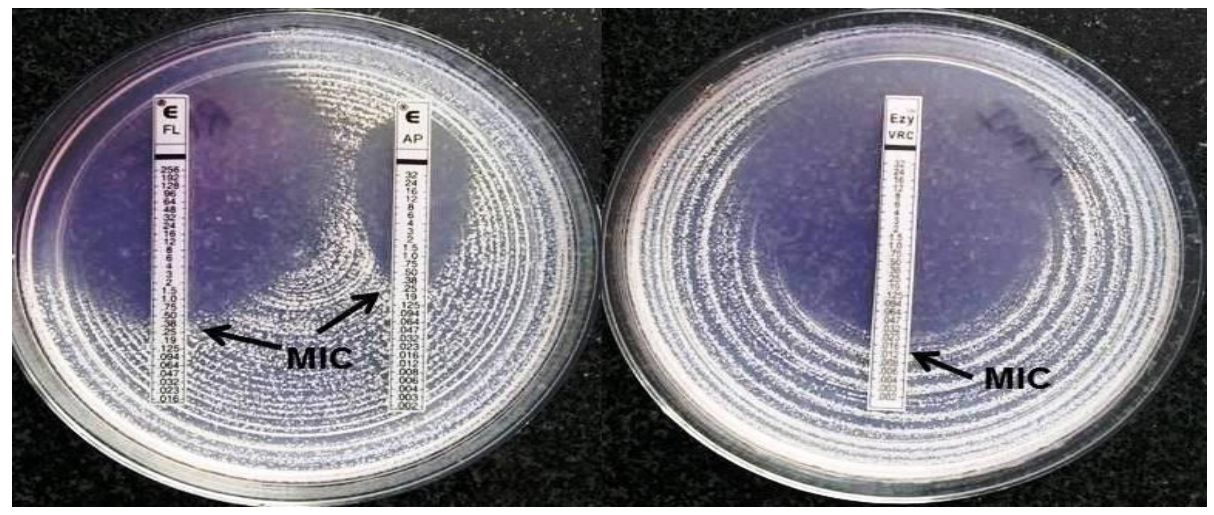

Fig.5 E-Test Method for AFST: Exhibiting Resistance to Fluconazole and Voriconazole

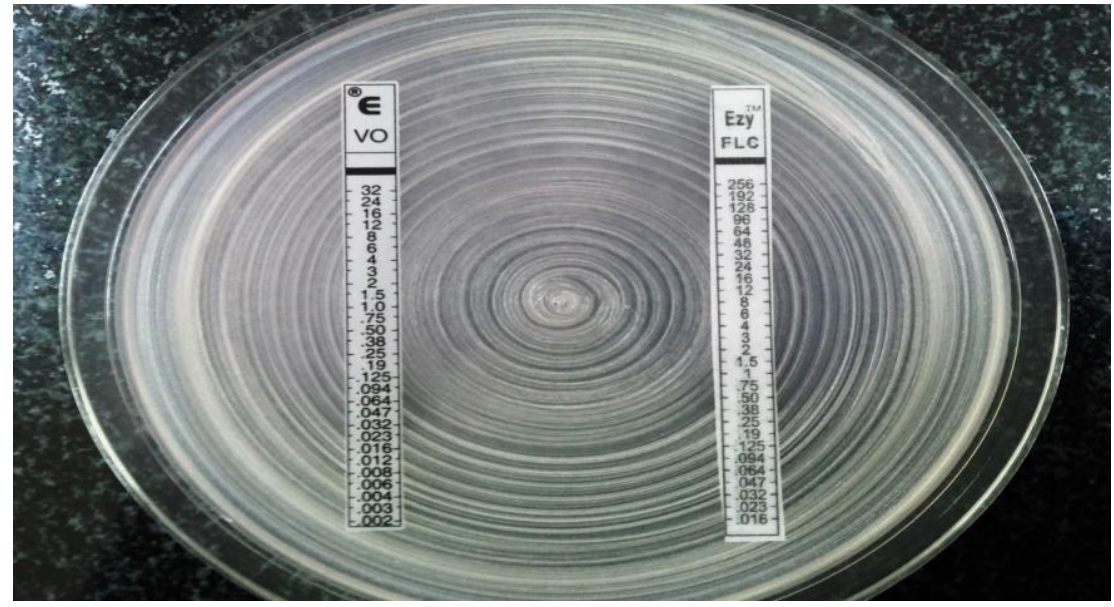

Fig.6 Distribution of Various Clinical Samples

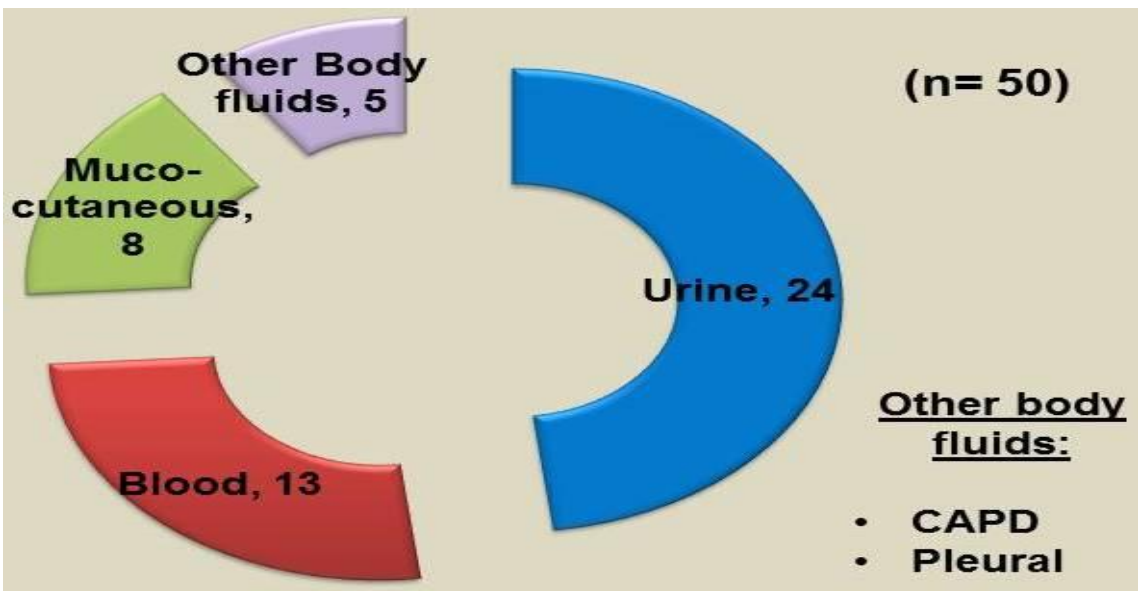


Fig.7 Distribution of Different Candida spp by Conventional Phenotypic Methods

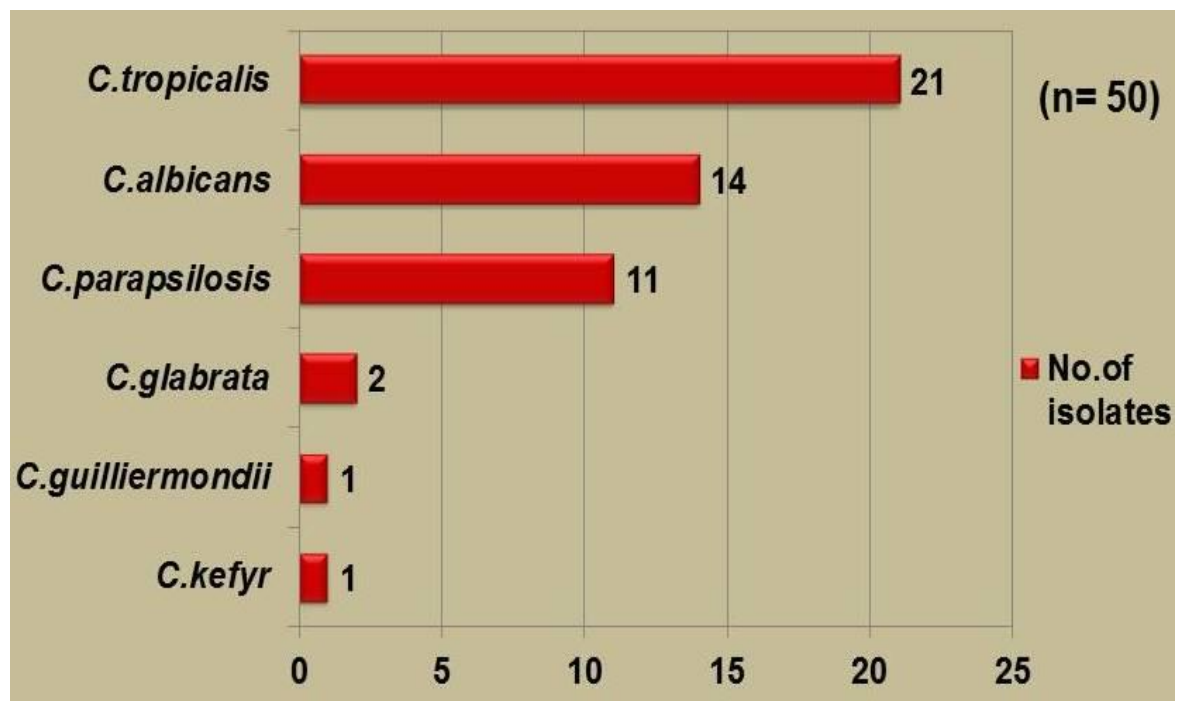

All the 50 isolates were subjected to antifungal susceptibility testing, out of which VITEK-2 identified only $4 \%$ (02 isolates) as resistant to fluconazole (C.guilliermondii and C.lusitaniae). By E-test method, $48 \%$ (24 isolates) of isolates were found to be resistant to fluconazole and voriconazole and all isolates were sensitive to amphotericin B (16).Out of these 24 resistant isolates, 14 were C.tropicalis, 05 were C.albicans, 04 were C.parapsilosis and 01 was C.glabrata. A total of $4 \%(02$ isolates) of isolates which showed fluconazole resistance by VITEK-2 were sensitive by E-test. Fluconazole and voriconazole resistance by E-test was $48 \%(24$ isolates) whereas VITEK-2 identified all of them as sensitive. There was less than chance agreement between VITEK-2 and E-test for antifungal susceptibility testing.

A study conducted by Charles $e t$ al reported the remarkable increase of inherently fluconazole resistant non-albicans Candida recently. They suggest that newer azoles like posaconazole and echinocandins are suitable alternatives (17).The fluconazole resistant
C.albicans, C.krusei and C.glabrata were found to be susceptible toVoriconazole invitro in a study by Barry AL et al (1996) and Ruhnke M et al (1997)(18,19). In this study, maximum fluconazole and voriconazole resistance was seen in C.tropicalis followed by C.albicans and others. These results are in concurrence with the results of other studies like Barry AL et al (1996), Ruhnke $\mathrm{M}$ et al (1997) and Barchiesi F et al (2000) $(18,19,20)$.In the present study, there was no isolate which was found to be resistant to amphotericin B, whereas some studies have reported a resistance of $6.9 \%$ byCapoor MRet al (2005) and 2.5 to $16.3 \%$ by Yang YL et al $(2005)(11,21)$.

In conclusion, in the present study, the prevalence of non-albicans Candida infection was more as compared to C.albicans and among the non-albicans Candida, C.tropicalis was most common species. We conclude that E-test method can be used as a simple method for antifungal susceptibility testing. Moreover, we cannot rely only on VITEK-2for identification and antifungal susceptibility testing. This study recommends the identification of Candida species by conventional methods and 
antifungal susceptibility testing by E-test method, because the treatment varies accordingly, due to the difference in antifungal susceptibility profile.

\section{References}

1. Jacqueline M, Achkar, Bettina Cet al. Candidal Infections of the genitourinary tract. Clinical Microbiology Review2010; 23(2):253-72.

2. Falgas ME, Apostolou KE, Pappas VD. Attributable mortality of Candidaemia: A systemic review of matched cohort and case control study. European Journal of clinical microbiology Infectious Disease 2006; 25:419-25.

3. Goodman JL, Winston DJ, Greenfield RA, Chandrasekar PH, Fox B, Kaiser H, et al. Acontrolled trial of fluconazole to prevent fungal infections in patients undergoingbone marrow transplantation. New England Journal of Medicine1992; 326:845-851.

4. Redding S, Smith J, Farinacci G, Rinaldi MG, Fothergill AWet al. Development of resistance tofluconazole among isolates of Candida albicans obtained during treatment of oropharyngeal candidiasis in AIDS: documentation by in vitro susceptibility testing and DNA subtype analysis. Journal of Clinical Infectious Disease 1994; 18:240-242.

5. Adhikary R, Joshi S. Species distribution and antifungal susceptibility of Candidaemia at multi super- speciality center in southern India. IJMM2011;29:309-11.

6. Chander J. A textbook of medical mycology (Mehta publishers, Nariana II, New Delhi, India). $3^{\text {rd }}$ edition 2009; 26683.

7. Topley WWC, Wilson CS. Candidiasis, in Topley Wilson's Medical mycology. Chapter 30:579- 13.
8. Rani R, Mohapatra NP, Mehta G, Randhawa VS. Changing trends of Candida species in tertiary north Indian hospital. Indian Journal of Medical Microbiology2002;20:42-4.

9. Agarwal J, Bansal S, Malik GK. Trends in neonatal septicemia. Emergence of non-albicans Candida, Indian $J$ Paediatrics 2004; 41:712-15

10. Chakrabarti A, Mohan B, Shrivastava SK, Marrak RSK, Ghosh A, Ray P. Change in distribution of Antifungal susceptibility of Candida species isolated from Candidaemia cases from tertriary centre during 1996-2000. Indian Journal of Medical Research2002;116:512.

11. Capoor MR, Nair D, Deb M, Verma PK, Srivastva L, Aggarwal P: Emergence of non-albicans Candida species and antifungal resistance in a tertiary care hospital. Japan Journal of Infectious Disease 2005; 58:344-48.

12. Krcmery V et al. non albicans Candida species causing fungaemia: pathogenicity and antifungal resistance. Journal of Hospital Infections 2002; 50 (4):243-260.

13. Wingard JR. Importance of Candida species other than C.albicans as pathogens in oncology patients. Clinical Infectious Disease 1995; 20:115-25.

14. Jensen RH, Arendrup MC. Characterization of a previously overlooked pathogen and its unique susceptibility profile in comparison with five related species. Journal of Clinical Microbiol2011;49:549-556.

15. Castanheira M, Woosley LN, Diekema DJ, Jones RN and Pfaller MA. Journal of Clinical Microbiology2013; 51(1):117.

16. Clinical and Laboratory Standards Institute (CLSI). Reference Method for Broth Dilution Antifungal Susceptibility Testing of Yeasts; Approved Standard. 
CLSI document M27-S4. Wayne, Pennsylvania, USA. 2012.

17. Charles PE, DoiseJM, Quenot JP, Aube $\mathrm{H}$, Dalle $\mathrm{F}$ et al.Candidaemia in critically ill patients: difference of outcome between medical and surgical patients. Intensive Care Medicine. 2003; 29:2162-2169.

18. Barry $\mathrm{AL}$ and Brown SD. In vitro studies of two triazole antifungal agents (voriconazole and fluconazole) against Candida species. Antimicrobial agents and chemotherapy 1996; 40:1948-9.

19. Ruhnke M, Schmidt-Westhausen A and Trautmann M. In-vitro activities of voriconazole against fluconazole susceptible and resistant C.albicans isolates from oral cavities of patients with HIV infection. Antimicrobial agents and chemotherapy1997; 41:575-7.
20. Barchiesi F, Arzeni D, Fothergill AW, Di Francesco LF, Caselli F et al. In vitro activities of the new antifungal triazole $\mathrm{SCH} 56592$ against common and emerging yeast pathogens. Antimicrob Agents Chemother2000; 44:226-229.

21. Yang YL, Li SY, Cheng HH and Lo HJ. Susceptibilities to amphotericin B and fluconazole of Candida species in TSRAY. Diagnostic Microbiology Infectious Disease 2005; 51:178-83.

22. Graph Pad Software Quickcalcs [home page on internet]. No date [cited 2014 Nov 20]. Available from: http://graphpad.com/ quickcalcs/ kappa1. cfm.

\section{How to cite this article:}

Prasanna, S., Partha Roy, Naveen Grover, Puneet Bhatt, Dudhat Vaibhav Laxmikant Rao and Nikunja Kumar Das. 2016. A Comparative Analysis on Identification and Antifungal Susceptibility Profile of Candida Isolates Using Conventional and Automated Techniques Int.J.Curr.Microbiol.App.Sci. 5(3): 579-589.doi: http://dx.doi.org/10.20546/ijcmas.2016.503.067 